\title{
Semi-global stabilization by an output feedback law from a hybrid state controller
}

\author{
Swann Marx ${ }^{\mathrm{a}}$, Vincent Andrieu ${ }^{\mathrm{b}}$ and Christophe Prieur ${ }^{\mathrm{a}}$ \\ ${ }^{a}$ GIPSA-lab, Department of Automatic Control, Grenoble Campus, 11 rue des Mathématiques, BP 46, 38402 Saint Martin \\ d'Hères Cedex. \\ ${ }^{\mathrm{b}}$ Université Lyon 1 CNRS UMR 5007 LAGEP, France and Fachbereich C - Mathematik und Naturwissenschaften, Bergische \\ Universität Wuppertal, Gaußstraße 20, 42097 Wuppertal, Germany.
}

\begin{abstract}
This article suggests a design method of a hybrid output feedback for SISO continuous systems. We focus on continuous systems for which there exists a hybrid state feedback law. A local hybrid stabilizability and a (global) complete uniform observability are assumed to achieve the stabilization of an equilibrium with a hybrid output feedback law. This is an existence result. Moreover, assuming the existence of a robust Lyapunov function instead of a stabilizability assumption allows to design explicitely this hybrid output feedback law. This last result is illustrated for linear systems with reset saturated controls.
\end{abstract}

Key words: Hybrid systems; Output feedback; Observers ; High-gain

\section{Introduction}

In recent years, many techniques for designing a stabilizing control law for nonlinear dynamical systems have been developed. It is now possible to achieve stabilization of equilibria for a large class of models. However, due to Brockett's necessary condition for stabilizability, it is well known that some systems cannot be stabilized by a continuous controller. Some of these systems can however be stabilized with a hybrid state feedback law, i.e. a discrete/continuous controller (see e.g. Prieur and Trélat (2006), where the Brockett integrator is stabilized with a quasi optimal hybrid control). Moreover, the use of hybrid control laws may be interesting to address performance issues (see e.g. Prieur (2001)). This explains the great interest of the control community in the synthesis of hybrid control laws (see Goebel et al. (2012), Hespanha et al. (2008); Hetel et al. (2013); Fichera et al. (2013); Yuan and Wu (2014)).

The output feedback stabilization problem has also attracted the attention of numerous researchers. Indeed, employing a state feedback law is most of the cases impossible, since the sensors can only access to partial mea-

Email addresses: marx.swann@gmail.com (Swann Marx), vincent.andrieu@gmail.com (Vincent Andrieu), christophe.prieur@gipsa-lab.fr (Christophe Prieur). surements of the state. Output feedback laws may be designed from a separation principle. More precisely, two tools are designed separately: a stabilizing state feedback law and an asymptotic state observer. However, if this approach is fruitful for linear systems, the separation principle does not hold in general for nonlinear systems. For instance, there exist stabilizable and observable systems for which the global asymptotic stabilization by output feedback is impossible (Mazenc et al. (1994)). Nevertheless, from weak stabilizability and observability assumptions, some semi-global results may be obtained (see e.g. Teel and Praly (1994) or (Isidori 1995, Pages 125-172)). However, in this case the observer and the state feedback have to be jointly designed (not separately) (see also Andrieu and Praly (2009) for some global results).

The aim of this paper is to address the stabilization by hybrid output feedback law. In Teel (2010), a local separation principle is stated. However, the construction of the observer is not explicit. Here, from a hybrid state feedback controller and an observability property, an algorithm is provided to build hybrid output feedback laws which stabilize semi-globally the equilibrium plant. If moreover a robust Lyapunov function is known, the feedback law design becomes explicit.

This article is organized as follows. Section 2 introduces 
the problem together with a hybrid stabilizability and a observability assumptions. The main result is given in Section 3.1. An equivalent stabilizability assumption in terms of Lyapunov function is considered in Section 3.2. This allows to give a more explicit theorem. Section 4 explains how to prove the first theorem from the second theorem. In Section 5 technical lemmas are stated in order to construct the suggested output feedback law. An illustrative example is given in Section 6. Finally, Section 7 collects some concluding remarks.

Note that this paper is an extension of the conference paper Marx et al. (2014). It includes a missing part (the observer design), proofs and a new illustration.

Notation: Given $\lambda \in \mathbb{N}, \mathbb{R}_{>\lambda}=[\lambda,+\infty)$. Given $n \in \mathbb{N}, I_{n} \in \mathbb{R}^{n \times n}$ denotes the identity matrix, i.e. $I_{n}=\operatorname{diag}(1, \ldots, 1)$. states for symmetric terms. Given $n \in \mathbb{N}, \mathcal{L}_{\text {loc }}^{\infty}\left(\mathbb{R}, \mathbb{R}^{n}\right)$ denotes the set of measurable locally bounded functions $u: \mathbb{R} \rightarrow \mathbb{R}^{n}$. A function $\alpha: \mathbb{R}_{\geq 0} \rightarrow \mathbb{R}_{\geq 0}$ belongs to class- $\mathcal{K}$ (for short $\alpha \in \mathcal{K}$ ) if it is continuous, zero at zero, and strictly increasing. A function $\beta: \mathbb{R}_{\geq 0} \times \mathbb{R}_{\geq 0} \rightarrow \mathbb{R}_{\geq 0}$ belongs to class- $\mathcal{K} \mathcal{L}$ (for short $\beta \in \mathcal{K} \mathcal{L})$ if it satisfies $(i)$ for each $t \geq 0, \beta(., t)$ is nondecreasing and $\lim _{t \searrow 0} \beta(s, t)=0$, and $(i i)$ for each $s \geq 0, \beta(s,$.$) is nonincreasing and \lim _{t \rightarrow \infty} \beta(s, t)=0$.

\section{Problem statement}

\subsection{Hybrid state feedback law for a continuous time plant}

The system under consideration is described by the following single-input single-output continuous dynamics:

$$
\dot{x}_{p}=f_{p}\left(x_{p}\right)+g_{p}\left(x_{p}\right) u, y=h_{p}\left(x_{p}\right),
$$

where $x_{p} \in \mathbb{R}^{n_{p}}, y \in \mathbb{R}, u \in \mathcal{U} \subset \mathbb{R}$. Note that $f_{p}$ : $\mathbb{R}^{n_{p}} \rightarrow \mathbb{R}^{n_{p}}$ and $g_{p}: \mathbb{R}^{n_{p}} \rightarrow \mathbb{R}^{n_{p}}, h_{p}: \mathbb{R}^{n_{p}} \rightarrow \mathbb{R}$ are $n_{p}+1$ times continuously differentiable ${ }^{1}, \mathcal{U}$ can be bounded (it yields a saturated control problems). Inspired by Prieur and Trélat (2006) and Sontag (1999), the origin, which is an equilibrium point for (1), is assumed to be stabilizable by a hybrid state feedback.

Assumption 1. (Persistent Flow Stabilizability) There exists a hybrid controller defined by $\left(\mathcal{F}_{c}, \mathcal{J}_{c}, f_{c}, g_{c}, \theta_{c}\right)$, where $\mathcal{F}_{c}$ and $\mathcal{J}_{c}$ are closed sets, $\mathcal{F}_{c} \cup \mathcal{J}_{c}=\mathbb{R}^{n_{p}+n_{c}}$, $g_{c}: \mathbb{R}^{n_{p}+n_{c}} \rightarrow \mathbb{R}^{n_{c}}, f_{c}: \mathbb{R}^{n_{p}+n_{c}} \rightarrow \mathbb{R}^{n_{c}}$ and $\theta_{c}:$ $\mathbb{R}^{n_{p}+n_{c}} \rightarrow \mathcal{U}$ are continuous functions and a positive value $\lambda$ in $(0,1)$ such that the set $\{0\} \times[0,1]$ in $\mathbb{R}^{n_{p}+n_{c}} \times \mathbb{R}$

\footnotetext{
1 These mappings are sufficiently smooth so that the mapping $\phi$ defined in (3) is $C^{1}$ and so that the function $B$ defined in (12) is locally Lipschitz.
}

is asymptotically stable for the system:

$$
\begin{aligned}
& \left\{\begin{array}{l}
\dot{x}_{p}=f_{p}\left(x_{p}\right)+g_{p}\left(x_{p}\right) \theta_{c}\left(x_{p}, x_{c}\right) \\
\dot{x}_{c}=f_{c}\left(x_{p}, x_{c}\right) \\
\dot{\sigma}=1-\sigma
\end{array} \quad\left(x_{p}, x_{c}, \sigma\right) \in \mathcal{F}_{c} \times \mathbb{R}_{\geq 0}\right. \\
& \left\{\begin{array}{l}
x_{p}^{+}=x_{p} \\
x_{c}^{+}=g_{c}\left(x_{p}, x_{c}\right) \\
\sigma^{+}=0
\end{array} \quad\left(x_{p}, x_{c}, \sigma\right) \in \mathcal{J}_{c} \times \mathbb{R}_{\geq \lambda} \quad(2 \mathrm{~b})\right.
\end{aligned}
$$

with basin of attraction $\mathcal{B} \times \mathbb{R}_{\geq 0}$, where $\mathcal{B}$ is an open subset of $\mathbb{R}^{n_{p}+n_{c}}$.

The sets $\mathcal{F}_{c} \times \mathbb{R}_{\geq 0}$ and $\mathcal{J}_{c} \times \mathbb{R}_{\geq \lambda}$ are called respectively the flow and jump sets associated to the continuous and discrete dynamics. The notion of solutions and of asymptotic stability discussed all along the paper are borrowed from Goebel et al. (2012).

Remark 1. An important feature of the hybrid state feedback control law is that its dynamics include a timer $\sigma$. It implies that there exists a dwell time between two consecutive jumps and consequently it prevents the existence of Zeno solutions. In the case in which this property is not satisfied for the state feedback, a timer can be added as presented in (Cai et al., 2008, Part V, C.). Such a technique is called a temporal regularization. However, in this case, only semi-global practical stability is obtained.

The problem under consideration in this paper is to design a stabilizing output feedback law based on this hybrid state feedback. The design presented in this paper requires an observability property for system (1) as described in the following section.

\subsection{Observability notions}

Following Gauthier et al. (1992), define the $C^{1}$ mapping $\phi: \mathbb{R}^{n_{p}} \rightarrow \mathbb{R}^{n_{p}}$ as follows

$$
\phi\left(x_{p}\right)=\left[h_{p}\left(x_{p}\right) L_{f_{p}} h_{p}\left(x_{p}\right) \ldots L_{f_{p}}^{n_{p}-1} h_{p}\left(x_{p}\right)\right]^{\top},
$$

where $L_{f_{p}}^{i} h_{p}(x)$ denotes the $i$-th Lie derivative of $h_{p}$ along $f_{p}$. The observability assumption employed all along the paper can be now stated.

Assumption 2 ((Global) Complete Uniform Observability (Gauthier et al. (1992))). System (1) is completely uniformly observable, that is

(i) The mapping $\phi: \mathbb{R}^{n_{p}} \rightarrow \phi\left(\mathbb{R}^{n_{p}}\right)=\mathbb{R}^{n_{p}}$ is a diffeomorphism;

(ii) System (1) is observable for any input $u(t)$, i.e. on any finite time interval $[0, T]$, for any measurable bounded input $u(t)$ defined on $[0, T]$, the initial state is uniquely determined on the basis of the output $y(t)$ and the input $u(t)$. 
Remark 2. In Marx et al. (2014), from a weaker observability assumption, i.e. an observability property holding for just one control, a finite-time convergent observer and a hybrid state feedback controller has been used to design an output feedback law. Such a strategy does not need a persistent flow stabilizability assumption. However only a weak stability property is obtained for the closed-loop system.

\section{Semi-global output feedback result}

\subsection{First main result}

Inspired by the approach of Teel and Praly (1994), from Assumptions 1 and 2, a semi-global output feedback result may be obtained.

Theorem 1 (Semi-global asymptotic stability). Assume Assumptions 1 and 2 hold. Assume moreover that $g_{c}$ satisfies that, for all $\left(x_{p}, x_{c}\right)$ in $\mathcal{B} \cap \mathcal{J}_{c}$, the set

$$
\left\{\left(x_{p}, g_{c}\left(w, x_{c}\right)\right), w \in \mathbb{R}^{n_{p}}\right\}
$$

is a compact subset of $\mathcal{B}$, then the origin of system (1) is semi-globally asymptotically stabilizable by a hybrid output feedback. In other words, for all compact sets $\Gamma$ contained in $\mathcal{B}^{p}:=\left\{x_{p} \in \mathbb{R}^{n_{p}},\left(x_{p}, 0\right) \in \mathcal{B}\right\}$, there exist a $C^{1}$ function $\Psi_{p}: \mathbb{R}^{n_{p}} \times \mathbb{R} \times \mathbb{R} \rightarrow \mathbb{R}$ and a positive real number $c_{x}$ such that the set $\{0\} \times[0,1]$ in $\mathbb{R}^{2 n_{p}+n_{c}} \times[0,1]$ is asymptotically stable for the system

$$
\begin{gathered}
\left\{\begin{array}{l}
\dot{x}_{p}=f_{p}\left(x_{p}\right)+g_{p}\left(x_{p}\right) u \\
\dot{\hat{x}}_{p}=\Psi_{p}\left(\hat{x}_{p}, y, u\right) \\
\dot{x}_{c}=f_{c}\left(\tilde{x}_{p}, x_{c}\right) \\
\dot{\sigma}=1-\sigma \\
y=h_{p}\left(x_{p}\right), u=\theta_{c}\left(\tilde{x}_{p}, x_{c}\right)
\end{array}\right. \\
\left(\tilde{x}_{p}, x_{c}, \sigma\right) \in \mathcal{F}_{c} \times \mathbb{R}_{\geq 0} \\
\left\{\begin{array}{l}
x_{p}^{+}=x_{p} \\
\hat{x}_{p}^{+}=\hat{x}_{p} \\
x_{c}^{+}=g_{c}\left(\tilde{x}_{p}, x_{c}\right) \\
\sigma^{+}=0
\end{array} \quad\left(\tilde{x}_{p}, x_{c}, \sigma\right) \in \mathcal{J}_{c} \times \mathbb{R}_{\geq \lambda} .\right.
\end{gathered}
$$

where $\tilde{x}_{p}$ is defined by 2

$$
\tilde{x}_{p}=\operatorname{sat}_{c_{x}}\left(\hat{x}_{p}\right)
$$

with basin of attraction containing $\Gamma \times\{0\} \times\{0\} \times \mathbb{R}_{\geq 0}$ (which is a subset of $\mathbb{R}^{n_{p}} \times \mathbb{R}^{n_{p}} \times \mathbb{R}^{n_{c}} \times \mathbb{R}_{\geq 0}$ ).

2 Given a positive real number $c$, sat s $_{c}: \mathbb{R}^{n} \rightarrow \mathbb{R}^{n}$ is the saturating vector function defined by $\operatorname{sat}_{c}(0)=0$ and $\operatorname{sat}_{c}(x):=x \min \left\{1, \frac{c}{|x|}\right\}, \forall x \neq 0$.
The design of the output feedback law which proves this theorem is based on a Lyapunov inverse theorem. However, two datas miss in the output feedback law given in Theorem 1: the positive real number $c_{x}$, the saturation level for the feedback law, and the observer dynamics $\Psi_{p}$. In order to give an explicit, the existence of a robust Lyapunov function is assumed, in the next section.

An important feature of this theorem is that an assumption needs to be imposed on the function $g_{c}$ (see equation (4)). This is due to a design of a set in which the solution should stay for a suitable duration. In the particular case in which $\mathcal{B}$ is $\mathbb{R}^{n_{p}+n_{c}}$, the previous condition is trivially satisfied if $g_{c}$ is such that

$$
\left|g_{c}\left(w, x_{c}\right)\right| \leq \gamma\left(\left|x_{c}\right|\right), \forall\left(w, x_{c}\right) \in \mathbb{R}^{n_{p}+n_{c}} \cap \mathcal{J}_{c}
$$

where $\gamma \in \mathcal{K}$. Moreover, note that there is a large class of systems that satisfy such a condition. For instance, switch systems or reset systems, as the one considered in Section 6 below.

\subsection{Second main result}

In this section an explicit result is introduced. It is based on the following assumption, where it is denoted $X=$ $\left[\begin{array}{lll}x_{p}^{\top} & x_{c}^{\top} & \sigma^{\top}\end{array}\right]^{\top}$.

Assumption 3 (Robust Lyapunov function). Let $\mathcal{B}$ be an open subset of $\mathbb{R}^{n_{p}} \times \mathbb{R}^{n_{c}}$ and let denote $\mathcal{A}:=\{0\} \times$ $[0,1] \subset \mathcal{B} \times \mathbb{R}_{>0}$. There exist a hybrid controller defined by $\left(\mathcal{F}_{c}, \mathcal{J}_{c}, f_{c}, g_{c}, \theta_{c}\right)$, where $\mathcal{F}_{c}$ and $\mathcal{J}_{c}$ are closed sets, $\mathcal{F}_{c} \cup \mathcal{J}_{c}=\mathbb{R}^{n_{p}+n_{c}}, g_{c}: \mathbb{R}^{n_{p}+n_{c}} \rightarrow \mathbb{R}^{n_{c}}, f_{c}: \mathbb{R}^{n_{p}+n_{c}} \rightarrow$ $\mathbb{R}^{n_{c}}$ and $\theta_{c}: \mathbb{R}^{n_{p}+n_{c}} \rightarrow \mathcal{U}$ are continuous functions, a positive value $\lambda$ in $(0,1)$, positive values $\alpha_{1}$ and $\alpha_{2} \in$ $(0,1)$ and a $C^{1}$ proper $\sqrt{3}$ function $V: \mathcal{B} \times \mathbb{R} \rightarrow \mathbb{R}_{\geq 0}$ satisfying $\{X \in \mathcal{B} \times \mathbb{R}, V(X)=0\}=\mathcal{A}$. For all positive real numbers $l$, the level set of $V$ defined as

$$
D_{l}:=\left\{\left(x_{p}, x_{c}, \sigma\right) \in \mathcal{B} \times \mathbb{R}_{\geq 0}: V\left(x_{p}, x_{c}, \sigma\right) \leq l\right\},
$$

is a compact subset of $\mathcal{B} \times \mathbb{R}$.

Moreover, there exists a positive real number $\varepsilon_{r}$ and an increasing $C^{0}$ function $\rho:\left[0, \varepsilon_{r}\right] \rightarrow \mathbb{R}_{+}$with $\rho(0)=0$ such that for all $(X, e)$ in $D_{l} \times \mathbb{R}^{n_{p}}$ such that $|e| \leq \varepsilon_{r}$, the following inequalities hold.

- If $\left(x_{p}+e, x_{c}, \sigma\right) \in\left(\mathcal{B} \cap \mathcal{F}_{c} \times \mathbb{R}_{\geq 0}\right)$

$\frac{\partial V}{\partial X}(X) F\left(X, x_{p}+e\right) \leq-\alpha_{1} V\left(x_{p}, x_{c}, \sigma\right)+\rho(|e|)$

- If $\left(x_{p}+e, x_{c}, \sigma\right) \in\left(\mathcal{B} \cap \mathcal{J}_{c} \times \mathbb{R}_{\geq \lambda}\right)$

$V\left(G\left(X, x_{p}+e\right)\right)-V(X) \leq-\alpha_{2} V\left(x_{p}, x_{c}, \sigma\right)+\rho(|e|)$ where $F$ is defined by

$F(X,):.=\left[\left(f_{p}\left(x_{p}\right)+g_{p}\left(x_{p}\right) \theta_{c}\left(., x_{c}\right)\right)^{\top} f_{c}\left(., x_{c}\right)^{\top} 1-\sigma\right]^{\top}$

3 A map is called proper if inverse images of compact sets are compact. 
and $G$ is defined by $G(X,):.=\left[\begin{array}{ll}x_{p}^{\top} & g_{c}\left(., x_{c}\right)^{\top} 0\end{array}\right]^{\top}$.

This assumption allows to obtain an explicit result.

Theorem 2 (Design of an output feedback law). Under Assumptions 2 and 3, assume that the set defined by (4) is a compact subset of $\mathcal{B}$. Then the set $\{0\} \times[0,1]$ in $\mathbb{R}^{n_{p}+n_{c}} \times \mathbb{R}$ is semi-globally asymptotically stabilizable. In other words, the conclusion of Theorem 1 holds. Moreover $c_{x}$ is computed in Section 5.1 and $\psi_{p}$ is computed in Section 5.2 from the Lyapunov function $V$ together with the robustness margin $\varepsilon_{r}$ and the positive value $\lambda$ of $A s$ sumption 3, and from the function $\phi$ of Assumption 2.

Let us note that it is difficult to be more explicit since the derivations of $c_{x}$ and $\Psi_{p}$ are quite long and require several steps. This is already the case in Teel and Praly (1994). Moreover, continuing what has been stated in Remark 1, it is crucial to have a Persistent-Flow Stabilizability in order to design explicitely our observer.

In a first step, $c_{x}$ is computed in (11) in order to force the solutions to remain in a compact set for a certain amount of (flow) time. The function $\Psi_{p}$ is a high-gain observer which is tuned in Lemma 2. It forces the error to reach the robustness margin obtained from Assumption 3 before the solution escapes the compact set.

The explicit construction of these two data and the proof that this output feedback law is a solution to Theorem 2 is reported in Section 5.

\section{Proof of Theorem 1 from Theorem 2}

In order to prove Theorem 1 from Theorem 2 it is sufficient to prove that Assumption 1 implies Assumption 3. This can be obtained from an inverse Lyapunov result. First, from (Goebel et al., 2012, Corollary 7.32) there exists a positive value $\alpha \in(0,1)$ and a smooth proper function $V: \mathcal{B} \times \mathbb{R} \rightarrow \mathbb{R}_{\geq 0}$ satisfying

$$
\begin{gathered}
\{X \in \mathcal{B} \times \mathbb{R}: V(X)=0\}=\mathcal{A} \\
\frac{\partial V}{\partial X}(X) F\left(X, x_{p}\right) \leq-V(X), \\
\forall\left(x_{p}, x_{c}, \sigma\right) \in\left(\mathcal{B} \cap \mathcal{F}_{c}\right) \times[0, \lambda] \\
V\left(G\left(X, x_{p}\right)\right)-V(X) \leq-\alpha V(X), \\
\forall\left(x_{p}, x_{c}, \sigma\right) \in\left(\mathcal{B} \cap \mathcal{J}_{c}\right) \times \mathbb{R}_{\geq 0}
\end{gathered}
$$

Let $l$ be a positive real number such that the level set $D_{l}$ is a compact subset of $\mathcal{B} \times \mathbb{R}$. Consider the two functions $r_{1}$ and $r_{2}$ defined as

$$
\begin{aligned}
r_{1}(s) & =\max _{|e| \leq s,\left(x_{p}+e, x_{c}, \sigma\right) \in D_{l}} \frac{\partial V(X)}{\partial X} F\left(X, x_{p}\right)+\frac{1}{2} V(X) \\
r_{2}(s) & =\max _{|e| \leq s,\left(x_{p}+e, x_{c}, \sigma\right) \in D_{l}} V\left(G\left(X, x_{p}\right)\right)-\left(1-\frac{1}{2} \alpha\right) V(X)
\end{aligned}
$$

Since $F, G$ are continuous and $V$ is smooth, $r_{1}$ and $r_{2}$ are also continuous functions. Moreover $r_{1}(0)<0$ and $r_{2}(0)<0$. Therefore there exist $\varepsilon_{r}^{1}$ and $\varepsilon_{r}^{2}$ such that $r_{1}(s)<0$ for all $s \leq \varepsilon_{r}^{1}$ and $r_{2}(s)<0$ for all $s \leq \varepsilon_{r}^{2}$. Let $\varepsilon_{r}=\min \left(\varepsilon_{r}^{1}, \varepsilon_{r}^{2}\right)$. For all $|e| \leq \varepsilon_{r}$ and $\left(x_{p}, x_{c}, \sigma\right) \in D_{l}$ it yields the following:

- If $\left(x_{p}+e, x_{c}, \sigma\right)$ in $\left(\mathcal{B} \cap \mathcal{F}_{c} \times \mathbb{R}_{\geq 0}\right)$,

$$
\frac{\partial V}{\partial X}(X) F\left(X, x_{p}\right) \leq-\frac{1}{2} V(X), \forall X \in \mathcal{B} \cap \mathcal{F}_{c} \times \mathbb{R}_{\geq 0}
$$

- If $\left(x_{p}+e, x_{c}, \sigma\right)$ in $\left(\mathcal{B} \cap \mathcal{J}_{c} \times \mathbb{R}_{\geq \lambda}\right)$

$V\left(G\left(X, x_{p}\right)\right)-V(X) \leq-\frac{1}{2} \alpha V(X), \forall X \in \mathcal{B} \cap \mathcal{J}_{c} \times \mathbb{R}_{\geq \lambda}$

Hence this Lyapunov function is the same than the one introduced in Assumption 3 with $\alpha_{1}=\frac{1}{2}$ and $\alpha_{2}=\frac{\alpha}{2}$.

Consider now the increasing function $\rho:\left[0, \varepsilon_{r}\right] \rightarrow$ $[0,+\infty)$ defined as follows 4

$$
\begin{array}{r}
\rho(s) \geq \max \left\{\begin{array}{c}
\max _{\left(x_{p}+e, x_{c}, \sigma\right) \in\left(D_{l_{4}} \cap \mathcal{F}_{c} \times \mathbb{R}_{\geq 0}\right),|e| \leq s} \nu_{1}(X, e), \\
\left.\max _{\left(x_{p}+e, x_{c}, \sigma\right) \in\left(D_{l_{4}} \cap \mathcal{J}_{c} \times \mathbb{R}_{\geq 0}\right),|e| \leq s} \nu_{2}(X, e)\right\}
\end{array}\right.
\end{array}
$$

where

$\nu_{1}(X, e)=\left|\frac{\partial V}{\partial X}(X)\left(F\left(X, x_{p}+e\right)-F\left(X, x_{p}\right)\right)\right|$,

and $\nu_{2}(X, e)=\left|V\left(G\left(X, x_{p}+e\right)\right)-V\left(G\left(X, x_{p}\right)\right)\right|$. With this function, Assumption 3 is satisfied. This ends the proof of Assumption 3 from Assumption 1. Therefore, as soon as Theorem 2 is valid, Theorem 1 holds under Assumptions 1 and 2.

\section{Construction of the output feedback law}

In the next sections, we follow a similar approach to Teel and Praly (1994). We first compute a saturation level $c_{x}$, a time of existence $T_{\min }$ and a compact subset of $\mathcal{B} \times \mathbb{R}_{\geq 0}$ denoted by $D_{l_{4}}$ such that, when saturating the controller with $c_{x}$, the solution starting from $\mathcal{B} \times \mathbb{R}_{\geq 0}$ remains in $D_{l_{4}}$ for all time less than $T_{\text {min }}$. Then, with $T_{\min }$ and the margin of robustness $c_{e}$ from Assumption 3, we design an observer such that the error dynamics converges to 0 asymptotically and such that, for all time higher than $T_{\min }$, the error dynamics belongs to the margin of robustness. Finally, we prove the attractiveness and the stability of the closed-loop system with the output feedback law.

$\overline{4}$ This function is well defined due to the fact that $\mathcal{F}_{c}$ and $\mathcal{J}_{c}$ are closed sets. 


\subsection{Selection of $c_{x}$ and minimmal time of existence of solutions}

This section differs from the strategy employed in Teel and Praly (1994). Indeed, since we have a hybrid dynamics, the solution of the closed-loop system can jump. Therefore, the computation of $T_{\min }$ becomes difficult. However, thanks to the timer dynamics, we can assert that between two jumps the solution of the closed-loop system belongs to the flow set. This allows us to compute $T_{\min }$ and thus $c_{x}$.

In the remaining part of this subsection, we consider the system defined by

$$
\begin{gathered}
\left\{\begin{array}{l}
\dot{x}_{p}=f_{p}\left(x_{p}\right)+g_{p}\left(x_{p}\right) \theta_{c}\left(\omega, x_{c}\right) \\
\dot{x}_{c}=f_{c}\left(\omega, x_{c}\right) \\
\dot{\sigma}=1-\sigma \\
\left(\omega, x_{c}, \sigma\right) \in \mathcal{F}_{c} \times \mathbb{R}_{\geq 0},
\end{array}\right. \\
\left\{\begin{array}{l}
x_{p}^{+}=x_{p} \\
x_{c}^{+}=g_{c}\left(\omega, x_{c}\right) \quad\left(\omega, x_{c}, \sigma\right) \in \mathcal{J}_{c} \times \mathbb{R}_{\geq \lambda}, \\
\sigma^{+}=0
\end{array}\right.
\end{gathered}
$$

where $\omega$ is an external perturbation function in $\mathbb{R}^{n_{p}}$. Such a system is not a classical hybrid system as the ones introduced in Goebel et al. (2012) since the flow and jump sets are defined with an external disturbance. Note that in the particular case in which $\omega=\tilde{x}_{p}$ defined in (6), the solution to system (10) by adding the dynamics of $\hat{x}_{p}$ is also solution to system (5). Hence, this implies the well-posedness of the closed-loop system as considered in (Goebel et al., 2012, Chapter 2).

Two cases may be distinguished to construct the sets: i) Solution to (10) does not jump; ii) Solution to (10) jumps at least one time. The first case is similar to the continuous case (and thus to Teel and Praly (1994)). The second case takes into account the hybrid behavior of the system under consideration. Under Assumption 3 , let $l_{1}=\max _{x_{p} \in \Gamma, \sigma \in[0, \lambda]} V\left(x_{p}, 0, \sigma\right)$. Note that $D_{l_{1}}$ is a compact subset of $\mathcal{B} \times \mathbb{R}_{>0}$ (see the notation employed in equation (7)). Let $l_{2}>l_{1}$ such that $D_{l_{2}} \subset \mathcal{B} \times \mathbb{R}_{\geq 0}$. To deal with the jump that can occur, we consider $D_{l_{2}}^{+}=$ $\bigcup_{\left(x_{p}, x_{c}, \sigma\right) \in D_{l_{2}}}\left\{\left(x_{p}, g_{c}\left(w, x_{c}\right), 0\right), w \in \mathbb{R}^{n_{p}}\right\}$. Since it is assumed that the set defined in (4) is a compact subset of $\mathcal{B} \times \mathbb{R}_{\geq 0}$, it yields that $D_{l_{2}}^{+}$is also a compact subset of $\mathcal{B} \times \mathbb{R}_{\geq 0}$. Let $l_{3}$ be such that $D_{l_{3}}$ is a compact subset which satisfies $D_{l_{2}}^{+} \subset D_{l_{3}} \subset \mathcal{B} \times \mathbb{R}_{\geq 0}$. Finally, let $l_{4}>l_{3}$ so that $D_{l_{4}}$ is a compact subset which contains $D_{l_{3}}$.

With these sets in hands, the positive real number $c_{x}$ can be selected as

$$
c_{x}=\max _{\left(x_{p}, x_{c}, \sigma\right) \in D_{l_{4}}}\left\{\left|x_{p}\right|\right\}
$$

Let us now establish the following property for solutions to system (10) initiated from $D_{l_{1}}$.

Lemma 1. (Minimal existence time of solution in $D_{l_{4}}$ ) There exists $T_{\min }>0$ such that for all $\omega$ in $\mathcal{L}_{\text {loc }}^{\infty}\left([0,+\infty) ; \mathbb{R}^{n_{p}}\right)$ with $|\omega(t)| \leq c_{x}$ for all $t$ in $\left[0, T_{\min }\right]$, and all $X^{\#}:=\left(x_{p}^{\#}, x_{c}^{\#}, \sigma^{\#}\right)$ in $D_{l_{1}}$, all solutions $x(\cdot, \cdot)$ to (10) with $X(0,0)=X^{\#}$ and all $(t, j)$ in $\operatorname{dom}(X)^{5}$ then $X(t, j) \in D_{l_{4}}$ for all $0 \leq t \leq T_{\min }$.

Proof. Let $\bar{V}$ the positive real number defined by $\bar{V}=\max _{X \in D_{l_{4}},|\omega| \leq c_{x}}\left|\frac{\partial V}{\partial X}(X) F(X, \omega)\right|$. In the remaining part of the proof, we show that Lemma 1 holds with $T_{\min }$ chosen as any positive real number satisfying

$$
T_{\min }<\min \left\{-\ln (1-\lambda), \frac{l_{2}-l_{1}}{\bar{V}}, \frac{l_{4}-l_{3}}{\bar{V}}\right\} \text {. }
$$

Let $X^{\#}$ be in $D_{l_{1}}$ and let $X$ be a solution to system (10) whose initial condition is $X^{\#}$. For all $(t, j)$ in $\operatorname{dom}(X)$. To ease the notation we denote $V(t, j)=V(X(t, j))$.

Let $(t, j)$ in $\operatorname{dom}(X)$ such that $0 \leq t \leq T_{\min }$. To prove the lemma, we need to show that $X(t, j)$ is in $D_{l_{4}}$. First of all, we show that $j \leq 1$. Indeed, assume $j \geq 2$. This implies that there exist $\bar{t}_{0}$ and $t_{1}$ such that $0 \leq t_{0}<t_{1} \leq t$ such that $\left(t_{0}, 0\right),\left(t_{0}, 1\right),\left(t_{1}, 1\right),\left(t_{1}, 2\right)$ are in $\operatorname{dom}(X)$. Note that $\sigma\left(t_{0}, 1\right)=0$ and $\sigma\left(t_{1}, 1\right)=\lambda$. Moreover, for all $s$ in $\left[t_{0}, t_{1}\right],(s, 1)$ is in $\operatorname{dom}(X)$ and $\dot{\sigma}(s, 1)=1-\sigma(s, 1)$. Hence, integrating this equation between $t_{0}$ and $t_{1}$, we get that $t \geq t_{1}-t_{0}>-\ln (1-\lambda) \geq T_{\min }$. This is impossible, and therefore $j \leq 1$.

So two cases may be distinguished.

$j=0$ This case is illustrated by Figure 1. $j=0$ implies that $s \in[0, t] \mapsto x(s, 0)$ is a continuous mapping with $x(0,0)$ in $D_{l_{1}} \subset D_{l_{2}}$. Hence we can define $t^{*}$, the largest time in $[0, t]$ such that $x(s, 0)$ is in $D_{l_{2}}$ (i.e. $t^{*}=\max _{s \in[0, t], x(\ell, 0) \in D_{l_{2}}, \forall \ell \in[0, s]}\{s\}$ ). Note that if $t^{*}=t$ then this implies that $x(t, 0)$ is in $D_{l_{2}}$, hence the result. Assume $t^{*}<t$. This implies that for all $s$ in $\left[0, t^{*}\right]$ we have $\dot{V}(s, 0)=\frac{\partial V}{\partial X}(X(s, 0)) F(X(s, 0), \omega(s)) \leq \bar{V}$. This gives $V\left(t^{*}, 0\right) \leq \bar{V} t^{*}+V(0,0) \leq \bar{V} T_{\min }+l_{1}<l_{2}$. Hence $x\left(t^{*}, 0\right)$ is in the interior of $D_{l_{2}}$. It yields that there exists $\varepsilon>0$ such that $x\left(t^{*}+\varepsilon, 0\right)$ is in the interior of $D_{l_{2}}$ which contradicts the fact that $t^{*}$ is an extremum.

$j=1$ This case is illustrated by Figure 2. $j=1$ implies that there exists $t_{0}$ in $[0, t]$ such that $\left(t_{0}, 0\right)$ and $\left(t_{0}, 1\right)$ are in $\operatorname{dom}(X)$ and $\left(w\left(t_{0}, 1\right), x_{c}\left(t_{0}, 1\right)\right)$ is in $\mathcal{J}_{c}$. Following the first case study, it is possible to show that $X\left(t_{0}, 0\right)$ is in $D_{l_{2}}$. Moreover,

5 The definition of $\operatorname{dom}(\mathrm{X})$ is borrowed from (Goebel et al. 2012, Definition 2.3). 


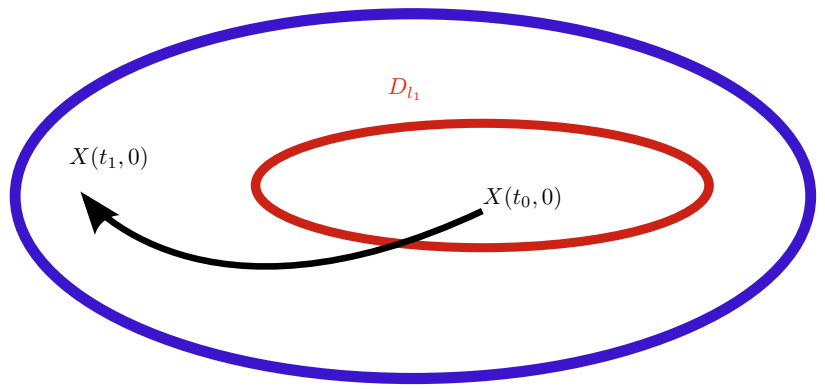

Fig. 1. Case 1: $j=0$

we have $x_{p}\left(t_{0}, 1\right)=x_{p}\left(t_{0}, 0\right)$ and $x_{c}\left(t_{0}, 1\right)=$ $g_{c}\left(w\left(t_{0}\right), x_{c}\left(t_{0}, 0\right)\right.$. This implies that $X\left(t_{0}, 1\right) \in D_{l_{2}}^{+}$. Note that $\left[t_{0}, t\right] \mapsto x(s, 1)$ is a continuous mapping with $X\left(t_{0}, 1\right)$ in $D_{l_{3}} \subset D_{l_{4}}$. As in the previous case, we define $t^{*}$, the largest time in $\left[t_{0}, t\right]$ such that $X(s, 1)$ is in $D_{l_{4}}$ (i.e. $\left.t^{*}=\max _{s \in\left[t_{0}, t\right], X(\ell, 1) \in D_{l_{4}}, \forall \ell \in\left[t_{0}, s\right]}\{s\}\right)$. Note that if $t^{*}=t$ then this implies that $X(t, 1)$ is in $D_{l_{4}}$, hence the result. Assume $t^{*}<t$. This implies that, for all $s$ in $\left[t_{0}, t^{*}\right]$, it holds $\dot{V}(s, 1)=\frac{\partial V}{\partial X}(X(s, 1)) F(X(s, 1), \omega(s)) \leq \bar{V}$. This implies $V\left(t^{*}, 1\right) \leq \bar{V} t^{*}+V\left(t_{0}, 1\right) \leq \bar{V} T_{\min }+l_{3}<l_{4}$. Hence $X\left(t^{*}, 1\right)$ is in the interior of $D_{l_{4}}$ and following the previous case, we get a contradiction.

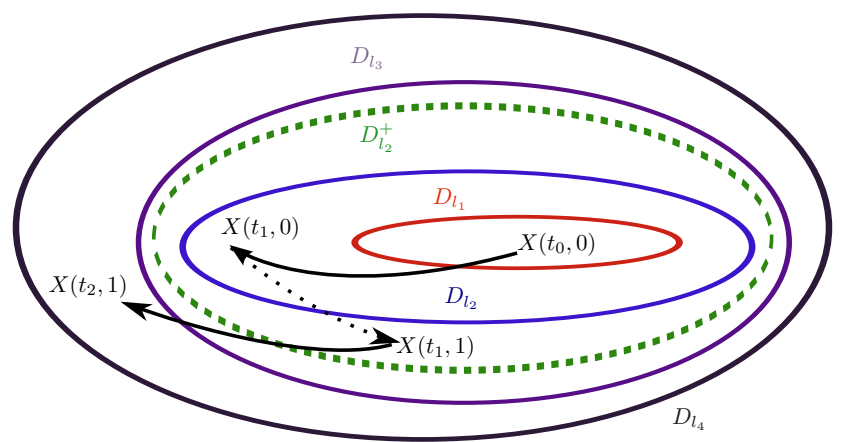

Fig. 2. Case 1: $j=1$. Continuous lines stand for flows. Dotted lines stand for jumps.

This concludes the proof of Lemma 1.

\subsection{Construction of $\Psi_{p}$}

In this subsection we design a high-gain observer for the system (1). Since only continuous time dynamics are considered in this subsection, to simplify the notation, only the flow components of the time domain are considered. The function $\Psi_{p}$ is selected to ensure that $\hat{x}_{p}$ estimates the state $x_{p}$ along solutions initiated in the projection of $D_{l_{1}}$ on $\mathbb{R}^{n_{p}}$ that is

$$
\begin{aligned}
\Pi_{p}\left(D_{l_{1}}\right)=\left\{x_{p} \in \mathbb{R}^{n_{p}}, \exists\left(x_{c}, \sigma\right) \in\right. & \mathbb{R}^{n_{c}} \times \mathbb{R}_{\geq 0}: \\
& \left.\left(x_{p}, x_{c}, \sigma\right) \in D_{l_{1}}\right\}
\end{aligned}
$$

and as long as these solutions remain in the projection of $D_{l_{4}}$ on $\mathbb{R}^{n_{p}}$ that is

$$
\begin{aligned}
\Pi_{p}\left(D_{l_{4}}\right)=\left\{x_{p} \in \mathbb{R}^{n_{p}}, \exists\left(x_{c}, \sigma\right) \in\right. & \mathbb{R}^{n_{c}} \times \mathbb{R}_{\geq 0}: \\
& \left.\left(x_{p}, x_{c}, \sigma\right) \in D_{l_{4}}\right\} .
\end{aligned}
$$

The estimation property has to be obtained for all input $u(\cdot)$ taking values in $\mathcal{U} \cap[-\bar{u}, \bar{u}]$ with $\bar{u}=\max _{\left|x_{p}\right| \leq c_{x},\left(x_{c}, \sigma\right) \in \Pi_{c}\left(D_{l_{4}}\right)} \theta_{c}\left(x_{p}, x_{c}\right)$ where

$$
\begin{aligned}
\Pi_{c}\left(D_{l_{4}}\right)=\left\{\left(x_{c}, \sigma\right) \in \mathbb{R}^{n_{c}+1}, \exists x_{p}\right. & \in \mathbb{R}^{n_{p}}: \\
& \left.\left(x_{p}, x_{c}, \sigma\right) \in D_{l_{4}}\right\},
\end{aligned}
$$

which is the projection of $D_{l_{4}}$ on $\mathbb{R}^{n_{c}}$. Moreover, the observer has to be designed such that the estimation error is smaller than the stability margin of the controller after $T_{\min }$. More precisely, with Assumption 3, and letting $l=l_{4}$ yields the positive real number $\varepsilon_{r}$ and the increasing mapping $\rho$. The observer has to be designed such that the set $\left\{\left(x_{p}, \hat{x}_{p}\right) \in \mathbb{R}^{2 n_{p}}:\left|x_{p}-\hat{x}_{p}\right|<c_{e}\right\}$, where $c_{e}:=\min \left\{\rho^{-1}\left(\frac{\alpha_{1} l_{4}}{2}\right), \rho^{-1}\left(\frac{\alpha_{2} l_{4}}{2}\right), \varepsilon_{r}\right\}$, is reached after $T_{\min }$.

A possible approach to design such observer is to use high-gain observer methodology. With Assumption 2, from (Gauthier et al., 1992, Theorem 2), setting $z=$ $\phi\left(x_{p}\right) \in \mathbb{R}^{n_{p}}$, the system (1) can be rewritten as follows:

$$
\dot{z}=A(z)+B(z) u
$$

with $z$ in $\mathbb{R}^{n_{p}}$ and

$$
A(z)=\frac{\partial \phi\left(x_{p}\right)}{\partial x_{p}} f_{p}\left(x_{p}\right)=\left[\begin{array}{llll}
z_{2} & \ldots & z_{n_{p}} & \chi(z)
\end{array}\right]^{\top}
$$

$$
\begin{aligned}
B(z) & =\frac{\partial \phi\left(x_{p}\right)}{\partial x_{p}} g_{p}\left(x_{p}\right) \\
& =\left[b_{1}\left(z_{1}\right) \ldots b_{n_{p}-1}\left(z_{1}, \ldots, z_{n_{p}-1}\right) b_{n_{p}}\left(z_{1}, \ldots, z_{n_{p}}\right)\right]^{\top} .
\end{aligned}
$$

where $\chi:=L_{f} h\left(\phi^{-1}(z)\right)$ and $b_{i}$ are locally Lipschitz functions as soon as $f_{p}$ is smooth enough. From there, the following lemma may be obtained.

Lemma 2. (Tunable observer) Let $\Psi_{p}: \mathbb{R}^{n_{p}} \times \mathbb{R} \times \mathbb{R} \rightarrow$ $\mathbb{R}^{n_{p}}$ be defined by

$$
\begin{aligned}
& \Psi_{p}\left(\hat{x}_{p}, y, u\right)=\left(\frac{\partial \phi}{\partial x_{p}}\left(\hat{x}_{p}, u\right)\right)^{-1} \\
& \quad\left[\tilde{A}\left(\phi\left(\hat{x}_{p}\right)\right)+\tilde{B}\left(\phi\left(\hat{x}_{p}\right)\right) u+\mathcal{L} K\left[y-h\left(\hat{x}_{p}\right)\right]\right]
\end{aligned}
$$


where $\tilde{A}: \mathbb{R}^{n_{p}} \rightarrow \mathbb{R}^{n_{p}}$ and $\tilde{B}: \mathbb{R}^{n_{p}} \rightarrow \mathbb{R}^{n_{p}}$ are functions

$$
\tilde{A}(z)=\left[\begin{array}{llll}
z_{2} & \ldots & z_{n_{p}} & \tilde{\chi}(z)
\end{array}\right]^{\top}
$$$$
\tilde{B}(z)=\left[\tilde{b}_{1}\left(z_{1}\right) \ldots \tilde{b}_{n_{p}-1}\left(z_{1}, \ldots, z_{n_{p}-1}\right) \tilde{b}_{n_{p}}\left(z_{1}, \ldots, z_{n_{p}}\right)\right]^{\top}
$$

and

$$
\mathcal{L}=\operatorname{diag}\left(\ell, \ldots, \ell^{n_{p}}\right)
$$

where $\ell$ is a positive real number larger than 1 and $K=$ $\left[\begin{array}{lll}-k_{1} & \cdots-k_{n_{p}}\end{array}\right]^{\top}$ is such that the matrix

$$
\mathcal{G}:=\left[\begin{array}{ccccc}
-k_{1} & 1 & 0 & \ldots & 0 \\
\vdots & 0 & 1 & \ddots & \vdots \\
\vdots & \vdots & \ddots & \ddots & 0 \\
\vdots & \vdots & & \ddots & 1 \\
-k_{n_{p}} & 0 & \ldots & \ldots & 0
\end{array}\right]
$$

is Hurwitz. Note that the functions $6 \tilde{b}_{i}: \mathbb{R}^{i} \rightarrow \mathbb{R}, i=$ $1, \ldots, n_{p}$ and $\tilde{\chi}: \mathbb{R}^{n_{p}} \rightarrow \mathbb{R}$ are such that

(1) For all $z$ in $\phi\left(D_{l_{4}}\right), \tilde{\chi}(z)=\chi(z)$ and $\tilde{b}_{i}(z)=b_{i}(z)$ where $\chi$ and $\left(b_{i}\right)$ 's are defined in (12);

(2) $\tilde{\chi}$ and $\tilde{b}_{i}$ are globally Lipschitz functions.

Moreover there exists a class $\mathcal{K} \mathcal{L}$ function $\beta$ such that for all solutions $x_{p}$ to (1) and all solutions $\hat{x}_{p}$ to

$$
\dot{\hat{x}}_{p}=\Psi_{p}\left(\hat{x}_{p}, y, u\right)
$$

with initial condition $\left(x_{p}^{\#}, \hat{x}_{p}^{\#}\right) \in \Pi_{p}\left(D_{l_{1}}\right) \times \Pi_{p}\left(D_{l_{1}}\right)$, input $u(\cdot)$ taking value in $\mathcal{U} \cap[-\bar{u}, \bar{u}]$, and for all t such that $x_{p}(s) \in \Pi_{p}\left(D_{l_{4}}\right)$, where $s \in[0, t]$, the following holds:

(1) $\left|x_{p}(s)-\hat{x}_{p}(s)\right| \leq \beta\left(\left|x_{p}^{\#}-\hat{x}_{p}^{\#}\right|, s\right), \forall s \in[0, t]$;

(2) If $t \geq T_{\min },\left|x_{p}(s)-\hat{x}_{p}(s)\right| \leq c_{e}, \forall s \in\left[T_{\min }, t\right]$.

Proof. There exists $c_{\chi}$ such that, for all $\left(z^{1}, z^{2}\right) \in$ $\left(\mathbb{R}^{n_{p}}\right)^{2},\left|\chi\left(z^{1}\right)-\chi\left(z^{2}\right)\right| \leq c_{\chi}\left|z^{1}-z^{2}\right|$. Note also that from the definition of $\phi$ we have $y=h_{p}\left(\phi\left(x_{p}\right)\right)=z_{1}$. With the Lipschitz property, the $\tilde{b}_{i}$ terms satisfy, for all $\left(z^{1}, z^{2}\right) \in \Pi_{p}\left(D_{l_{4}}\right)^{2},\left|\tilde{b}_{i}\left(z^{1}\right)-\tilde{b}_{i}\left(z^{2}\right)\right| \leq c_{b_{i}} \sum_{j=1}^{i}\left|z_{j}^{1}-z_{j}^{2}\right|$. Moreover, setting $\hat{z}:=\phi\left(\hat{x}_{p}\right)$, it yields along the solutions to (16):

$$
\dot{\hat{z}}=\tilde{A}(\hat{z})+\tilde{B}(\hat{z}) u+\mathcal{L} K\left[y-\hat{z}_{1}\right],
$$

As standard in high-gain observer design, let $\tilde{e}$ be the scaled error components:

$$
\tilde{e}_{i}=\frac{\hat{z}_{i}-\left(\phi\left(x_{p}\right)\right)_{i}}{\ell^{i-1}} .
$$

\footnotetext{
6 These functions exist from the Kirszbraun extension theorem, see e.g. (Federer, 1969, Definition 2.10.43) and from the fact that $\phi\left(\bar{D}_{l_{4}}\right)$ is a compact subset of $\mathbb{R}^{n_{p}}$.
}

Therefore, as soon as $x_{p} \in \Pi_{p}\left(D_{l_{4}}\right)$, it holds

$$
\dot{\tilde{e}}=\ell \mathcal{G} \tilde{e}+\Delta\left(\hat{z}, x_{p}, u\right)
$$

where $\Delta\left(\hat{z}, x_{p}, u\right)$ is obtained from $\tilde{\chi}$ and $\tilde{b}_{i}$ for all $i=$ $1, \ldots, n_{p}$. From the structure of the observation error, there exists a positive real number $c_{\Delta}$ such that for all $\left(\hat{z}, x_{p}, u\right)$ in $\mathbb{R}^{n_{d}} \times \Pi_{p}\left(D_{l_{4}}\right) \times \mathcal{U} \cap[-\bar{u}, \bar{u}]$ and for all $\ell>1$ $\left|\Delta\left(\hat{z}, x_{p}, u\right)\right| \leq c_{\Delta}|\tilde{e}|$. The matrix $\mathcal{G}$ being Hurwitz, there exist a positive definite matrix $P$ and a positive value $d$ satisfying

$$
P \mathcal{G}+\mathcal{G}^{\top} P \leq-d P .
$$

Let $U: \mathbb{R}^{n_{p}} \rightarrow \mathbb{R}_{\geq 0}$ be the following (Lyapunov) function $U(\tilde{e})=\tilde{e}^{\top} P \tilde{e}$. Its time derivative along the solutions to the system (19) with $\left(\hat{z}, x_{p}, u\right)$ in $\mathbb{R}^{n_{d}} \times \Pi_{p}\left(D_{l_{4}}\right) \times \mathcal{U} \cap$ $[-\bar{u}, \bar{u}]$ and $\ell>1$ satisfies the following inequalities.

$$
\begin{aligned}
\dot{U}(\tilde{e}) & \leq-\ell d U(\tilde{e})+2 \tilde{e}^{\top} P \Delta\left(\hat{z}, x_{p}, u\right) \\
& \leq-\ell d U(\tilde{e})+2 c_{\Delta}|P \tilde{e}||\tilde{e}| \\
& \leq-\ell d U(\tilde{e})+2 c_{\Delta} \frac{\lambda_{\max }(P)}{\lambda_{\min }(P)} U(\tilde{e})
\end{aligned}
$$

Consider a solution $x_{p}$ to (1) and a solution $\hat{x}_{p}$ to (16) with initial condition $\left(x_{p}^{\#}, \hat{x}_{p}^{\#}\right) \in \Pi_{p}\left(D_{l_{1}}\right)^{2}$, input $u(\cdot)$ taking value in $\mathcal{U} \cap[-\bar{u}, \bar{u}]$, and $t$ such that $x_{p}(s)$ is in $\Pi_{p}\left(D_{l_{4}}\right)$ for all $s$ in $[0, t]$. With Grönwall lemma, we get along this solution

$$
U(\tilde{e}(t)) \leq \exp \left(-\left(\ell d-2 c_{\Delta} \frac{\lambda_{\max }(P)}{\lambda_{\min }(P)}\right) t\right) U(\tilde{e}(0))
$$

Moreover, for all $\left(x_{p}, \hat{z}\right)$, it holds that

$$
\left|\phi\left(x_{p}\right)-\hat{z}\right|^{2} \frac{\lambda_{\min }(P)}{\ell^{2 n_{p}}} \leq U(\tilde{e}) \leq \frac{\lambda_{\max }(P)}{\ell^{2}}\left|\phi\left(x_{p}\right)-\hat{z}\right|^{2},
$$

hence,

$$
\begin{aligned}
\left|\phi\left(x_{p}(t)\right)-\hat{z}(t)\right| & \leq \exp \left(-\left(\frac{\ell}{2} d-c_{\Delta} \frac{\lambda_{\max }(P)}{\lambda_{\min }(P)}\right) t\right) \\
\ell^{n_{p}-1} & \frac{\lambda_{\max }(P)}{\lambda_{\min }(P)}\left|\phi\left(x_{p}^{\#}\right)-\phi\left(\hat{x}_{p}^{\#}\right)\right| .
\end{aligned}
$$

Note that $\Pi_{p}\left(D_{l_{4}}\right)$ and $\phi\left(\Pi_{p}\left(D_{l_{4}}\right)\right)$ are compact subsets of $\mathbb{R}^{n_{p}}$ and that the functions $\phi: \mathbb{R}^{n_{p}} \rightarrow \mathbb{R}^{n_{p}}$ and $\phi^{-1}: \mathbb{R}^{n_{p}} \rightarrow \mathbb{R}^{n_{p}}$ are continuous. Hence, the two class $\mathcal{K}$ functions $\nu$ and $\nu^{*}$ can be defined as

$$
\begin{array}{cr}
\nu(s) & =\max _{x_{p} \in \Pi_{p}\left(D_{l_{4}}\right),\left|x_{p}^{*}-x_{p}\right| \leq s}\left|\phi\left(x_{p}\right)-\phi\left(x_{p}^{*}\right)\right| \\
\nu^{*}(s) & =\max _{z \in \phi\left(\Pi_{p}\left(D_{l_{4}}\right)\right),\left|z^{*}-z\right| \leq s}\left|\phi^{-1}(z)-\phi^{-1}\left(z^{*}\right)\right| .
\end{array}
$$


These functions satisfy that, for all $z$ in $\phi\left(\Pi_{p}\left(D_{l_{4}}\right)\right)$ and $\hat{z}$ in $\mathbb{R}^{n_{p}}$,

$$
\begin{aligned}
\left|\phi^{-1}(z)-\phi^{-1}(\hat{z})\right| & \leq \nu^{*}(|z-\hat{z}|) \\
|z-\hat{z}| & \leq \nu\left(\left|\phi^{-1}(z)-\phi^{-1}(\hat{z})\right|\right) .
\end{aligned}
$$

Let now $\ell>1$ be any positive real number such that

$$
\begin{gathered}
\Omega(\ell):=\frac{\ell}{2} d-c_{\Delta} \frac{\lambda_{\max }(P)}{\lambda_{\min }(P)}>0 \\
\nu\left(\exp \left(-\Omega(\ell) T_{\min }\right) \ell^{n_{p}-1} \mathfrak{M}\right) \leq c_{e},
\end{gathered}
$$

where $\mathfrak{M}:=2 \frac{\lambda_{\max }(P)}{\lambda_{\min }(P)} \max _{z \in \phi\left(\Pi_{p}\left(D_{l_{1}}\right)\right)}|z|$. We can rewrite (26) as follows:

$\exp \left(-\ell \frac{T_{\min } d}{2}\right) \exp \left(T_{\min } c_{\Delta} \frac{\lambda_{\max }(P)}{\lambda_{\min }(P)}\right) \ell^{n_{p}-1} \leq \frac{\nu^{-1}\left(c_{e}\right)}{\mathfrak{M}}$

Since the exponential function dominates the polynomial function, the condition (26) is satisfied, for $\ell$ sufficiently large. From (23), (24) and (25) the first item of Lemma 2 holds with the function

$$
\beta(s, t)=\nu^{*}\left(\exp (-\Omega(\ell) t) \ell^{n_{p}-1} \frac{\lambda_{\max }(P)}{\lambda_{\min }(P)} \nu(s)\right) .
$$

The second item of Lemma 2 is deduced from (23), (24) and (26) and the fact that $\left(x_{p}^{\#}, \hat{x}_{p}^{\#}\right) \in \Pi_{p}\left(D_{l_{1}}\right) \times$ $\Pi_{p}\left(D_{l_{1}}\right)$.

\subsection{Proof of Theorem 2}

Consider the positive value $c_{x}$ obtained in (11) and the function $\Psi_{p}$ obtained in (13). In the first part of the proof, we show attractivity of the set $\{0\} \times[0,1]$ in $\mathbb{R}^{2 n_{p}+n_{c}} \times[0,1]$ along the solutions to system (5) whose initial condition is in $\tilde{\Gamma}:=\Gamma \times\{0\} \times \mathbb{R}_{\geq 0} \subset \mathcal{B}^{p} \times\{0\} \times$ $\mathbb{R}_{\geq 0}$. The stability is shown in a second part.

Note that the hybrid system (5) satisfies the basic assumptions for hybrid systems (Goebel et al., 2009, Assumption 6.5.) with the flow set $\mathcal{F}_{c}^{o}=\left\{\left(x_{p}, \hat{x}_{p}, x_{c}, \sigma\right) \in\right.$ $\left.\mathbb{R}^{2 n_{p}+n_{c}} \times \mathbb{R}_{\geq 0}: \mathfrak{F}\left(x_{p}, \hat{x}_{p}, x_{c}, \sigma\right) \in \mathbb{R}^{n_{p}} \times \mathcal{F}_{c} \times \mathbb{R}_{\geq 0}\right\}$ and the jump set $\mathcal{J}_{c}^{o}=\left\{\left(x_{p}, \hat{x}_{p}, x_{c}, \sigma\right) \in \mathbb{R}^{2 n_{p}+n_{c}} \times\right.$ $\left.\mathbb{R}_{\geq 0}: \mathfrak{F}\left(x_{p}, \hat{x}_{p}, x_{c}, \sigma\right) \in \mathbb{R}^{n_{p}} \times \mathcal{J}_{c} \times \mathbb{R}_{\geq \lambda}\right\}$, where $\mathfrak{F}:$ $\left(x_{p}, \hat{x}_{p}, x_{c}, \sigma\right) \mapsto\left(x_{p}, \operatorname{sat}_{c_{x}}\left(\hat{x}_{p}\right), x_{c}, \sigma\right)$.

First Part : Attractivity. Let $X^{\#}:=\left(x_{p}^{\#}, x_{c}^{\#}, \hat{x}_{p}^{\#}, \sigma^{\#}\right)$ be in $\tilde{\Gamma}$ and consider a solution $X:=\left(x_{p}, x_{c}, \hat{x}_{p}, \sigma\right)$ to (5) whose initial condition is $X^{\#}$ and defined on its time domain denoted $\operatorname{dom}(X)$.

Note that the system (5) can be rewritten as the hybrid system (10) with $\omega=\operatorname{sat}_{c_{x}}\left(\hat{x}_{p}\right)$ and $\hat{x}_{p}$ is given with the observer (13).
With Lemma 1 and with the $\sigma$ dynamics (persistent flow), we know that there exists $j_{0}$ such that $\left(T_{\min }, j_{0}\right)$ is in $\operatorname{dom}(X)$ and for all $(t, j)$ in $\operatorname{dom}(X)$ with $t \leq T_{\min }$ then $\left(x_{p}(t, j), x_{c}(t, j), \sigma(t, j)\right)$ is in $D_{l_{4}}$. Thus, for all $(t, j)$ in $\operatorname{dom}(X)$ with $t \leq T_{\min }$, the control input satisfies $|u(t, j)| \leq \bar{u}$. Let $\operatorname{dom}_{D_{l_{4}}}(X)$ be the time domain of the solution restricted to the set $D_{l_{4}}$. With Lemma 2, for all $(t, j)$ in $\operatorname{dom}_{D_{l_{4}}}(X)$ with $t \geq T_{\min },\left|x_{p}(t, j)-\hat{x}_{p}(t, j)\right| \leq$ $c_{e}$. Moreover, for all $(t, j)$ in $\operatorname{dom}_{D_{l_{4}}}(X)$ with $t \geq T_{\min }$, we have $\left|x_{p}(t, j)\right| \leq c_{x}$. It implies

$$
\begin{aligned}
\left|x_{p}(t, j)-\tilde{x}_{p}(t, j)\right| & =\left|\operatorname{sat}_{c_{x}}\left(x_{p}(t, j)\right)-\operatorname{sat}_{c_{x}}\left(\hat{x}_{p}(t, j)\right)\right| \\
& \leq\left|x_{p}(t, j)-\hat{x}_{p}(t, j)\right| \leq c_{e} .
\end{aligned}
$$

It is now possible to show that for all $(t, j)$ in $\operatorname{dom}(X)$, $X(t, j)$ is in $D_{l_{4}}$. We will argue by contradiction to prove this assertion. By assuming that it is false, two cases may occur.

- The solution escapes $D_{l_{4}}$ when flowing. Hence, there exists $\left(t_{0}, j_{0}\right)$ in $\operatorname{dom}(X)$ such that $\left(x_{p}, x_{c}, \sigma\right)\left(t_{0}, j_{0}\right)$ is in $D_{l_{4}}$ and for all $\varepsilon>0$, there exists $\delta<\varepsilon$ such that $\left(t_{0}+\delta, j_{0}\right)$ is in $\operatorname{dom}(X)$ and $\left(x_{p}, x_{c}, \sigma\right)\left(t_{0}+\delta, j_{0}\right)$ is not in $D_{l_{4}}$. Note that this implies that $\left(x_{p}, x_{c}\right)\left(t_{0}, j_{0}\right)$ is at the boundary of $D_{l_{4}}$. Consequently, this implies $V\left(t_{0}, j_{0}\right)=$ $l_{4}$. Note moreover, keeping in mind that $\mid x_{p}\left(t_{0}, j_{0}\right)-$ $\tilde{x}_{p}\left(t_{0}, j_{0}\right)|\leq| x_{p}\left(t_{0}, j_{0}\right)-\hat{x}_{p}\left(t_{0}, j_{0}\right) \mid \leq c_{e} \leq \varepsilon_{r}$ we get, from Assumption 3, $\dot{V}\left(t_{0}, j_{0}\right) \leq-\alpha_{1} V\left(t_{0}, j_{0}\right)+\rho\left(c_{e}\right) \leq$ $-\frac{\alpha_{1} l_{4}}{2}$. This implies that the function $s \mapsto V\left(t_{0}+s, j_{0}\right)$ is strictly decreasing. It contradicts the existence of small $\varepsilon$.

- The solution escapes $D_{l_{4}}$ when jumping. Hence, there exists $\left(t_{0}, j_{0}\right)$ in $\operatorname{dom}(X)$ such that $\left(x_{p}, x_{c}, \sigma\right)\left(t_{0}, j_{0}\right)$ is in $D_{l_{4}}$ and $\left(x_{p}, x_{c}, \sigma\right)\left(t_{0}, j_{0}+1\right)$ is not in $D_{l_{4}}$. Since $\left|x_{p}\left(t_{0}, j_{0}\right)-\hat{x}_{p}\left(t_{0}, j_{0}\right)\right| \leq c_{e} \leq \varepsilon_{r}$, with Assumption 3, it follows $V\left(t_{0}, j_{0}+1\right) \leq\left(1-\alpha_{2}\right) V\left(t_{0}, j_{0}\right)+\rho\left(c_{e}\right) \leq$ $\left(1-\frac{\alpha_{2}}{2}\right) l_{4}<l_{4}$. This is a contradiction with the escape of the solution from $D_{l_{4}}$.

Consequently, for all $(t, j)$ in $\operatorname{dom}(X)$, we have $x(t, j)$ is in $D_{l_{4}}$. Note that the timer forces the $t$ direction of the time domain to be unbounded. Hence, thanks to the Lemma $2, \lim _{t+j \rightarrow+\infty}\left|\hat{x}_{p}(t, j)-x_{p}(t, j)\right|=0$. We get the result employing the triangular structure of the system with the ISS property in $D_{l_{4}}$ (i.e. Assumption 3) (see e.g. (Cai and Teel, 2009, Theorem 3.1.)).

Second Part: Stability. To conclude the proof, let us prove the stability property. Let $S_{\varepsilon}$ be defined by $S_{\varepsilon}=$ $\left\{\left(x_{p}, x_{c}, \hat{x}_{p}, \sigma\right): V\left(x_{p}, x_{c}, \sigma\right) \leq \varepsilon\right.$ and $\left.\left|x_{p}-\hat{x}_{p}\right| \leq \varepsilon\right\}$, where $\varepsilon<l_{1}$. Moreover, let $\mathcal{N}_{l}$ be an open neighborhood of $\mathcal{A}$ defined as

$$
\begin{aligned}
& \mathcal{N}_{l}=\left\{\left(x_{p}, x_{c}, \hat{x}_{p}, \sigma\right): V\left(x_{p}, x_{c}, \sigma\right)\right)<\frac{\varepsilon}{4}, \\
& \left.\beta\left(\left|x_{p}-\hat{x}_{p}\right|, 0\right)<\min \left\{\rho^{-1}\left(\frac{\alpha_{1} \varepsilon}{4}\right), \rho^{-1}\left(\frac{\alpha_{2} \varepsilon}{4}\right), \frac{\varepsilon}{2}\right\}\right\},
\end{aligned}
$$


where $(\beta, \rho)$ and $\left(\alpha_{1}, \alpha_{2}\right)$ are the two functions and the two positive real numbers given in Lemma 2 and Assumption 3. Note that $\mathcal{N}_{\ell} \subset \mathcal{S}_{\varepsilon}$.

Now consider $X$ a solution to the closed-loop system (5) starting from any point of $\mathcal{N}_{l}$ with time domain denoted $\operatorname{dom}(X)$. Let $\operatorname{dom}_{S_{\varepsilon}}(X)$ denote the hybrid time domain of the solution restricted to the set $S_{\varepsilon}$.

Let us show that this solution $X$ remains in $\mathcal{S}_{\varepsilon}$. Note that $\hat{x}_{p}(0,0)$ and $x_{p}(0,0)$ are in $\Pi_{p}\left(D_{l_{1}}\right)$. With Lemma 2 , it yields that for all $(t, j)$ in $\operatorname{dom}_{S_{\varepsilon}}(X)$,

$$
\begin{aligned}
\left|x_{p}(t, j)-\hat{x}_{p}(t, j)\right| & \leq \beta\left(\left|x_{p}(0,0)-\hat{x}_{p}(0,0)\right|, 0\right) \\
& \leq \min \left\{\rho^{-1}\left(\frac{\alpha_{1} \varepsilon}{4}\right), \rho^{-1}\left(\frac{\alpha_{2} \varepsilon}{4}\right), \frac{\varepsilon}{2}\right\} .
\end{aligned}
$$

Moreover, $\left(x_{p}(t, j), x_{c}(t, j), \sigma(t, j)\right)$ is in $D_{l_{1}} \subset D_{l_{4}}$, hence from the ISS inequalities that hold in $D_{l_{4}}$ (see Assumption 3), we get for all $t_{2}>t_{1}$ and $j$ such that $\left(t_{1}, j\right)$ and $\left(t_{2}, j\right)$ are in $\operatorname{dom}_{S_{\varepsilon}}(X)$

$$
\begin{aligned}
& \frac{d}{d t} V\left(x_{p}(t, j), x_{c}(t, j), \sigma(t, j)\right) \\
& \quad \leq-\alpha_{1} V\left(x_{p}(t, j), x_{c}(t, j), \sigma(t, j)\right)+\frac{\alpha_{1} \varepsilon}{4}
\end{aligned}
$$

which implies with $V\left(X\left(t_{1}, j\right)\right) \leq \frac{\varepsilon}{4}$

$$
V\left(x_{p}(t, j), x_{c}(t, j), \sigma(t, j)\right) \leq \frac{\varepsilon}{4}, t \in\left[t_{1}, t_{2}\right]
$$

Hence, it can not escape $S_{\varepsilon}$ by flowing since it does not reach the boundary of $S_{\varepsilon}$. Moreover, for all $(t, j)$ in $\operatorname{dom}_{S_{\varepsilon}}(X)$ such that $(t, j+1)$ is in $\operatorname{dom}(X)$, from the ISS inequalities of Assumption 3 once again, it yields, if $V(X(t, j)) \leq \frac{\varepsilon}{4}$

$$
\begin{aligned}
& V\left(x_{p}(t, j+1), x_{c}(t, j+1), \sigma(t, j+1)\right) \leq \\
& \quad\left(1-\alpha_{2}\right) V\left(x_{p}(t, j), x_{c}(t, j), \sigma(t, j)\right)+\frac{\alpha_{2} \varepsilon}{4}<\frac{\varepsilon}{4}
\end{aligned}
$$

Hence, $X(t, j+1) \in S_{\varepsilon}$.

Thus, if the initial condition $X^{\#}$ is in $\mathcal{N}_{l}$, then the solution remains in $S_{\varepsilon}$. Since $S_{\varepsilon}$ can be made as closed as wanted from $\mathcal{A}$, this proves stability of the set $\mathcal{A}$ and concludes the proof of Theorem 1 .

\section{Application to a First Order Reset Element (FORE) system}

To illustrate Theorem 1, the following second order system is considered:

$$
\dot{x}_{p}=\left[\begin{array}{ll}
0 & 1 \\
1 & 0
\end{array}\right] x_{p}+\left[\begin{array}{c}
-1 \\
0
\end{array}\right] \operatorname{sat}_{u_{0}}(v), \quad y=\left[\begin{array}{ll}
1 & 0
\end{array}\right] x_{p} .
$$

With $u=\operatorname{sat}_{u_{0}}(v), \mathcal{U}=\left[-u_{0}, u_{0}\right]$ and $u_{0}=3$, it is a system of the form (1). Note that the set of control $\mathcal{U}$ is bounded and that $y=x_{p_{1}}$. Consequently, despite the fact that the dynamics are linear, the stabilization problem for this system is not an easy task although recent works Yuan and $\mathrm{Wu}(2015)$; Hu et al. (2006) propose design methods for saturated actuator. The strategy presented in this paper can be also efficient for purely nonlinear systems as it is shown in Marx et al. (2014) where it is proven the existence of a hybrid output feedback for a chain of integrators with a nonlinearity from a state feedback uniting a global and a local controllers. Following Loquen et al. (2007), we compute the hybrid controller such that $\mathcal{A}=\{0\} \times\{0\} \times\{0\} \times[0,1]$ is locally asymptotically stable for the FORE system

$$
\left\{\begin{aligned}
\dot{x}_{p}= & {\left[\begin{array}{ll}
0 & 1 \\
1 & 0
\end{array}\right] x_{p}+\left[\begin{array}{c}
-1 \\
0
\end{array}\right] \text { sat }_{u_{0}}\left(k x_{c}\right) } \\
\dot{x}_{c}= & {\left[\begin{array}{cc}
-2 & 0 \\
0 & -12
\end{array}\right] x_{c}-x_{p} } \\
\dot{\sigma}= & 1-\sigma \\
& \left(x_{p}^{\top} M x_{c} \geq 0\right) \text { and } \sigma \in \mathbb{R}_{\geq 0}
\end{aligned}\right.
$$

$$
\left\{\begin{array}{l}
x_{p}^{+}=x_{p} \\
x_{c}^{+}=0 \\
\sigma^{+}=0
\end{array} \quad\left(x_{p}^{\top} M x_{c} \leq 0\right) \text { and } \sigma \in \mathbb{R}_{\geq \lambda}\right.
$$

with $k=[-13-30], \lambda=0.01, M=\left[\begin{array}{cc}0 & -I_{1} \\ -I_{1} & 0\end{array}\right]$ and

$\mathcal{E}\left(P_{V}\right)=\left\{\left(x_{p}, x_{c}\right) \in \mathbb{R}^{4},\left[x_{p} ; x_{c}\right] P_{V}\left[x_{p} ; x_{c}\right]^{\top} \leq 1\right\} \subset \mathcal{B}$

where $P_{V}$ is the positive definite matrix defined as $P_{V}=$ $\left[\begin{array}{llll}4.0560 & 0.6868 & 6.6342 & 0.0000 \\ * & 6.4905 & 0.0000 & 0.6342\end{array}\right]$

$\left.\begin{array}{cccc}* & 6.4905 & 0.0000 & 6.6342 \\ \star & \star & 49.4184 & 6.6162\end{array}\right]$. The matrix $P_{V}$ has been computed to satisfy a set of linear matrix inequalities (LMIs) given in (Loquen et al., 2007, Theorem 3.) employing the LMI solver Sturm (1999). Note that employing an equivalent continuous feedback law $u_{c}=$ sat $_{u_{0}}\left(k x_{c}\right)$, without any reset, leads to a smaller estimation of the basin of attraction (i.e. the matrix $P_{V}^{c}$ that describes the lower approximation of the basin of attraction has bigger eigenvalues than $P_{V}$ ). The set $\mathcal{B}$ denotes the basin of attraction and $\mathcal{E}\left(P_{V}\right)$ is a lower approximation of this one. Moreover, letting $\alpha_{1}=0.4017, \alpha_{2}=0.6078$, $\varepsilon_{r}=0.0550$ and the function $\rho(|e|):=13.3418|e|$, Assumption 3 is satisfied.

Since (27) satisfies the well-known Kalman observability rank condition for the pair $(C, A)$, Assumption 2 holds for the sytem (27). Moreover, denoting the projection $\mathcal{E}\left(P_{V}\right)$ on $\mathbb{R}^{n_{p}}$ by $\Pi_{p}\left(\mathcal{E}\left(P_{V}\right)\right)=\left\{x_{p} \in \mathbb{R}^{2}: 4.0560 x_{p_{1}}^{2}+\right.$ $\left.6.4905 x_{p_{2}}^{2}+1.3736 x_{p_{1}} x_{p_{2}} \leq 1\right\}$ and letting $\Gamma=\left\{x_{p} \in\right.$ $\left.\mathbb{R}^{2}: 4.0560 x_{p_{1}}^{2}+6.4905 x_{p_{2}}^{2}+1.3736 x_{p_{1}} x_{p_{2}} \leq 0.6\right\} \subset$ $\Pi_{p}\left(\mathcal{E}\left(P_{V}\right)\right)$, hence, it is obtained from Theorem 1 that 
the set $\{0\} \times\{0\} \times[0,1]$ is asymptotically stable for the sytem (28) with basin of attraction containing $\Gamma \times\{0\} \times$ $\{0\} \times \mathbb{R}_{\geq 0}$.

Following Lemmas 1 and 2, we aim at computing all the variables. Here, from the definition of $\Gamma$, we let $l_{1}=0.6$ and $l_{2}=0.7$. Due to $(28), g_{c}=0$ and thus $D_{l_{2}}^{+} \subset D_{l_{2}}$. It follows that $l_{3}=0.8$ and $l_{4}=0.9$ are suitable values. Thus, we can compute $c_{x}:=\sqrt{P^{-1 / 2} Q P^{-1 / 2} l_{4}}=$ 0.6624 where $Q=\operatorname{diag}(1,1,0,0)$. We check that $k_{1}=$ -6 and $k_{2}=-9$ are such that $\mathcal{G}$ defined in Lemma 2 is Hurwitz. With all these parameters, we numerically compute $c_{e}=0.0136, T_{\min }=0.01, d=2, P=$ $\left[\begin{array}{lll}0.1000 & 0.0077 \\ 0.0077 & 0.0635\end{array}\right]$ and $\mathfrak{M}=1.2070$. Then we check that $\ell=1000$ satisfies (26). Finally we can build the observer satisfying the structure of (13). Therefore we can apply Theorem 2 and state that closing the loop of (27) with the observer of the form (16) and the output feedback law coming from (28) leads to a semi-global stability.

With $\left(x_{p}^{\#}, x_{c}^{\#}, \hat{x}_{p}^{\#}, \sigma^{\#}\right)=(0.15,0,0,0,0,0,0) \subset \mathcal{E}(P) \times$ $[0,1]$, The time evolution of $x_{p_{1}}$ and $\hat{x}_{p_{1}}$ satisfying respectively (27) and (16) are given by Figure 3 . The first figure illustrates the convergence of the observer, the second the asymptotic stability of the closed-loop system. Figure 4 illustrates the phase portrait of $x_{p}$.
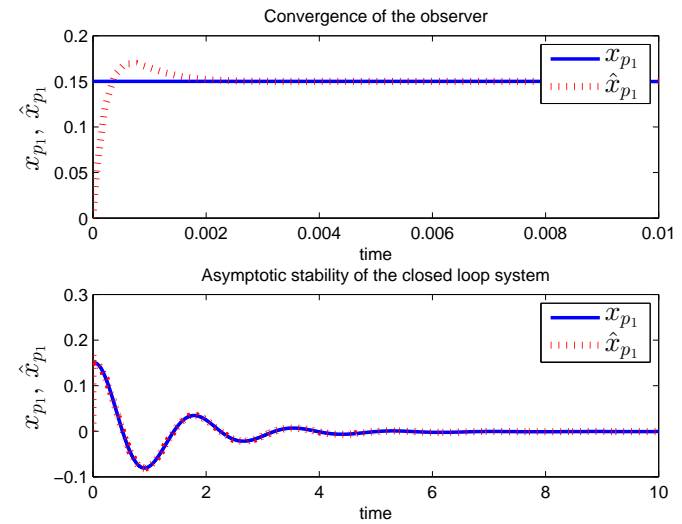

Fig. 3. Time evolution of $x_{p_{1}}$ (blue plain line) and $\hat{x}_{p_{1}}$ (red dashed line).

\section{Conclusion}

An output feedback law has been designed for SISO affine systems for which there exists a hybrid state feedback under an observability assumption. Some special assumptions (for instance the existence of a timer) have been useful to deal with the hybrid case. The result has been applied to design a hybrid output feedback law from a FORE controller.

Some open questions follow. Does an output feedback exist even with a less strong assumption than the Assumption 1, i.e. an assumption which does not state any

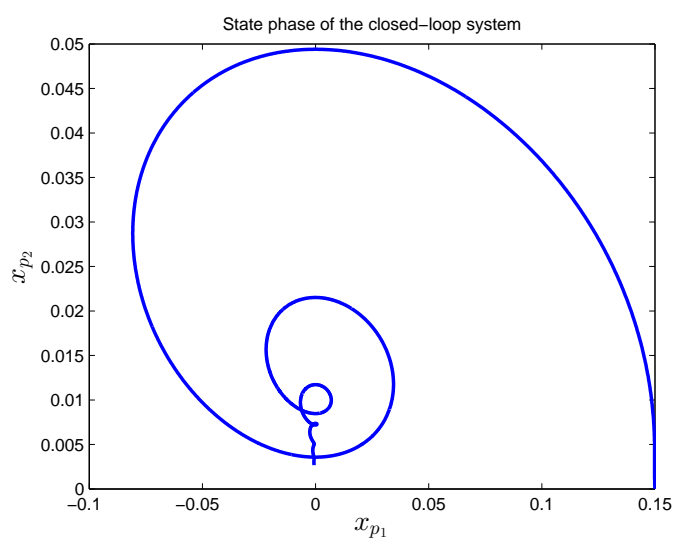

Fig. 4. Phase portrait of the state $x_{p}$.

knowledge of the time between two consecutive jumps? Can the design method be applied on systems for which it does not exist a continuous state feedback such as the Arstein circle? In Andrieu and Praly (2009), some output feedback laws are presented to achieve global stabilization of equilibrium point. Assuming stronger observability property, can such output feedback laws be achieved for hybrid case? Moreover, designing an observer that converges in finite time with a stability property, and thus adapting Andrieu et al. (2008) for the hybrid systems case, could be also a interesting research line.

\section{References}

Andrieu, V. and Praly, L. (2009). A unifying point of view on output feedback designs for global asymptotic stabilization. Automatica, 45, 1789-1798.

Andrieu, V., Praly, L., and Astolfi, A. (2008). Homogeneous approximation, recursive observer design, and output feedback. SIAM Journal of Control and Optimization, 47, 1814-1859.

Cai, C., Teel, A.R., and Goebel, R. (2008). Smooth Lyapunov functions for hybrid systems part II : (pre)asymptotically stable compact sets. IEEE Trans. Automat. Control, 53, 734-748.

Cai, C. and Teel, A. (2009). Characterizations of inputto-state stability for hybrid systems. Systems $\&$ Control Letters, 58, 47-53.

Federer, H. (1969). Geometric Measure Theory. Springer-Verlag Berlin.

Fichera, F., Prieur, C., Tarbouriech, S., and Zaccarian, L. (2013). Using Luenberger observers and dwell-time logic for feedback hybrid loops in continuous-time control systems. International Journal of Robust and Nonlinear Control, 23(10), 1065-1086.

Gauthier, J.P., Hammouri, H., and Othman, S. (1992). A simple observer for nonlinear systems. applications to bioreactors. IEEE Trans. on Automat. Control, 37, 875-880.

Goebel, R., Sanfelice, R., and Teel, A. (2009). Hybrid 
dynamical systems. Control Systems Magazine, 29, 28-93.

Goebel, R., Sanfelice, R., and Teel, A. (2012). Hybrid Dynamical Systems: modeling, stability, and robustness. Princeton University Press.

Hespanha, J.P., Liberzon, D., and Teel, A. (2008). Lyapunov conditions for input-to-state stability for impulsive systems. Automatica, 44(11), 2735-2744.

Hetel, L., Daafouz, J., Tarbouriech, S., and Prieur, C. (2013). Stabilization of linear impulsive systems through a nearly-periodic reset. Nonlinear Analysis: Hybrid Systems, 7(1), 4-15.

Hu, T., Teel, A., and Zaccarian, L. (2006). Stability and performance for saturated systems via quadratic and nonquadratic Lyapunov functions. IEEE Trans. Automat. Control, 51(11), 1770-1786.

Isidori, A. (1995). Nonlinear control systems, volume 1. Springer Science \& Business Media.

Loquen, T., Tarbouriech, S., and Prieur, C. (2007). Stability analysis for reset systems with input saturation. In 46th Conference on Decision and Control, 32723277. New Orleans, LA.

Marx, S., Andrieu, V., and Prieur, C. (2014). Using a high-gain observer for a hybrid output feedback: finitetime and asymptotic cases for SISO affine systems. In American Control Conference, 4637-4642. Portland, OR.

Mazenc, F., Praly, L., and Dayawansa, W. (1994). Global stabilization by output feedback : Examples and Counter-Examples. Systems \& Control Letters, $23,67-78$.

Prieur, C. (2001). Uniting local and global controllers with robustness to vanishing noise. Mathematics of Control, Signals, and Systems, 14(2), 143-172.

Prieur, C. and Trélat, E. (2006). Quasi-optimal robust stabilization of control systems. SIAM Journal on Control and Optimization, 45(5), 1875-1897.

Sontag, E. (1999). Stability and stabilization: discontinuities and the effect of disturbances. In Nonlinear analysis, differential equations and control, 551-598. Springer Netherlands.

Sturm, J.F. (1999). Using SeDuMi 1.02, a Matlab toolbox for optimization over symmetric cones. Optimization Methods and Software, 11(1-4), 625-653.

Teel, A. (2010). Observer-based hybrid feedback: a local separation principle. In American Control Conference, 898-903. Baltimore, MD.

Teel, A. and Praly, L. (1994). Global stabilizability implies semi-global stabilizability by output feedback. Systems \&3 Control Letters, 22, 313-325.

Yuan, C. and Wu, F. (2014). Analysis and synthesis of linear hybrid systems with state-triggered jumps. Nonlinear Analysis: Hybrid Systems, 14, 47-60.

Yuan, C. and Wu, F. (2015). Switching control of linear systems subject to asymmetric actuator saturation. International Journal of Control, 88(1), 204-215. 